\title{
ADAPTIVE OUTPUT FEEDBACK CONTROL OF A SCALE HELICOPTER RESTRICTED TO A 2DOF PLATFORM
}

\author{
J. C. Raimúndez ${ }^{* *}$ J. L. Camaño ${ }^{* *}$ M. Béjar * \\ J. A. Baltar** \\ ${ }^{*}$ ETSII, Universidad de Sevilla, SPAIN \\ ${ }^{* *}$ ETSII, Universidade de Vigo, SPAIN
}

\begin{abstract}
In this paper we present the application of the adaptive neural-networktechnique in (Calise, Hovakimyan and Idam, 2001) to the control of the 3DoF model helicopter in (Avila, Brogliato, Dzul and Lozano, 2003). The technique uses feedback linearization, linear (coarse) compensation and adaptive-neural-network (fine) compensation. The description of the technique is presented in a way as self-contained as possible. The helicopter model also includes a one-sided constraint to address take-off and landing, as well as hover. Our study shows that a very simple and poor plant model is adequate, and that the the neural network compensates this, completely on-line, without initial training. Copyright $(2005$ IFAC.
\end{abstract}

Keywords: Neural networks, output feedback control, nonlinear control, adaptive control.

\section{INTRODUCTION}

The growing interest in adaptive techniques with output feedback stems in the good response to control, needed in modern applications involving complex systems. Complex modelling, appears when using actuators and observers with strong nonlinear interaction with the plant. In this situation the model lacks precision to attend implementation of high performance controllers. Examples can be found in areas such as: flexible structures, fluid mechanics, combustion processes, aeronautics, etc.

Synthesis approaches to adaptive output feedback control typically make use of state estimation (Seshagiri and Khalil, 2000), (Ge, Hang and Zhang, 1999), and therefore require plant dimensions knowledge. Some approaches further restrict the output to have full relative degree.

1 This work was supported by CICYT, under project DPI 200204401-C03-03.
The presented technique (Hovakimyan and Calise, 2002) removes all these restrictions by adopting a direct output feedback approach that does not rely on state estimation. The design employs plant relative order knowledge, coupled with an on-line radial basis neural network (NN) to compensate for modeling errors.

A linear dynamic compensator is designed to stabilize the linearized plant. A signal, comprised of a linear combination of the measured tracking error and the compensator states, is used to adapt the $\mathrm{NN}$ weights. The input vector to the NN is composed by current and past input/output data.

The control system is augmented by a low-pass filter designed to satisfy a strictly positive real (SPR) condition of a transfer function associated with the Lyapunov stability analysis, which is used to construct the $\mathrm{NN}$ adaptation law using only available measurement as a training signal, and to prove boundedness of all the error signals of the closed loop system. 
The adaptive technique is applied in the present paper to the design of a tracking controller for a scale model benzin helicopter (VARIO) mounted in a 2DOF platform as described in (Avila, Brogliato, Dzul and Lozano, 2003).

The content of the paper is as follows. In section 2, we present the generic plant, and in section 3, we describe the design process. The details of the technique are presented in a way as self contained as possible. In section 4 the main theorem on tracking error boundedness is recalled, for completeness. Section 5 introduces the helicopter model, the control solution that we have developed and simulations. Advantages are shown in section 6 .

\section{PROBLEM STATEMENT}

Let the dynamics of an observable and stabilizable nonlinear multi-input multi-output (MIMO) system be given by the following set of equations:

$$
\begin{aligned}
& \dot{x}=f(x, u) \\
& y=h(x)
\end{aligned}
$$

when $x \in \Omega \subset \mathbb{R}^{n}$ is the system state ( $n$ unknown but finite), $u, y \in \mathbb{R}^{m}$ are control and measurement signals respectively, $f(\cdot, \cdot), h(\cdot) \in \mathcal{C}^{\infty}$ are unknown functions.

Assumption 1a Let the dynamics of (1) satisfy the conditions for output feedback linearization with vector relative degree $\mathbf{r}=\left(r_{1}, r_{2}, \cdots, r_{m}\right)$ with $r_{1}+$ $r_{2}+\cdots+r_{m} \leq n$. Then there exists a mapping $\xi=\Phi(x)$, where

$$
\Phi(x)=\left[\begin{array}{c}
\phi_{1} \\
\phi_{2} \\
\vdots \\
\phi_{m}
\end{array}\right], \phi_{i}=\left[\begin{array}{c}
L_{f}^{0} h_{i} \\
L_{f}^{1} h_{i} \\
\vdots \\
L_{f}^{\left(r_{i}-1\right)} h_{i}
\end{array}\right]
$$

with $L_{f}^{(i)} h_{k}$ being the Lie derivatives, $h_{k}$ the $h$ components in (1), that transforms (1) into

$$
\begin{aligned}
\dot{\eta} & =f_{0}(\xi, \eta) \\
\dot{\xi}_{i}^{1} & =\xi_{i}^{2} \\
& \vdots \\
\dot{\xi}_{i}^{r_{i}} & =g_{i}(\xi, \eta, u) \\
\dot{\xi}_{i}^{1} & =y_{i}, \quad\{i=1, \cdots, m\}
\end{aligned}
$$

the so called normal form, where $g_{i}=L_{f}^{r_{i}} h_{i}, \xi=$ $\left(\xi_{1}^{\top}, \cdots, \xi_{m}^{\top}\right)^{\top}, \xi_{i}=\left(\xi_{i}^{1}, \cdots, \xi_{i}^{r_{i}}\right)^{\top}$ and $\eta$ are the internal dynamic states.

Assumption 1b The system has asymptotic stable internal dynamics, defined in (3) when $\xi=0$.

\section{CONTROLLER DESIGN}

In this section, a summary of the technique (Calise, Hovakimyan and Idam, 2001) is presented.
Feedback Linearization. Is performed by introducing the transformation

$$
\hat{\nu}=\hat{g}(y, u)
$$

where $\hat{g}$ is invertible with respect to $u$. $\hat{g}$ is any available approximation of $g=\left(g_{1}, \cdots, g_{m}\right)^{\top}$ in (3). Introducing $\nu=\left(\nu_{1}, \cdots, \nu_{m}\right)^{\top}$ as a pseudo control, results

$$
u=\hat{g}^{-1}(y, \nu)
$$

Introducing $y^{(r)}=\left(y_{1}^{\left(r_{1}\right)}, \cdots, y_{m}^{\left(r_{m}\right)}\right)^{\top}$ and considering

$$
\begin{aligned}
\Delta(x, \nu) & =\Delta(\xi, \eta, \nu) \\
& =g\left(\xi, \eta, \hat{g}^{-1}(y, \nu)\right)-\hat{g}\left(y, \hat{g}^{-1}(y, \nu)\right)
\end{aligned}
$$

the system dynamics can be reduced to

$$
y^{(r)}=\nu+\Delta
$$

where $\Delta$ is the difference between $g(\xi, \eta, u)$ and its approximation $\hat{g}(y, u)$. Defining the tracking error as

$$
e=\tilde{y}=y_{c}-y
$$

where $y_{c}=\left(y_{c_{1}}, \cdots, y_{c_{m}}\right)^{\top}$ are references to follow by the outputs, the pseudo control can be chosen as

$$
\nu=y_{c}^{(r)}+\nu_{d c}-\nu_{a d}
$$

where $\nu_{d c}$ is a dynamic linear compensator output and $\nu_{a d}$ is the adaptive control element output designed to cancel $\Delta$. Then the dynamics in (7) reduce to

$$
y^{(r)}=y_{c}^{(r)}+\nu_{d c}-\nu_{a d}+\Delta
$$

As shown in (6), $\Delta$ depends on $\nu_{a d}$ through $\nu$ while $\nu_{a d}$ has to be designed to cancel $\Delta$. In order to guarantee the existence and uniqueness of a solution to $\nu_{a d}$ is introduced the next assumption.

Assumption 2 The mapping $\nu_{a d} \rightarrow \Delta$ is a contraction over the entire input domain of interest. A contraction is defined by the condition:

$$
\left\|\frac{\partial \Delta}{\partial \nu_{a d}}\right\|<1
$$

Applying this condition to (6)

$$
\left\|\frac{\partial \Delta}{\partial \nu_{a d}}\right\|=\left\|\frac{\partial(g-\hat{g})}{\partial u} \frac{\partial u}{\partial \hat{g}}\right\|<1
$$

which after simplification can be represented as

$$
\left\|\frac{\partial g}{\partial u}\left(\frac{\partial \hat{g}}{\partial u}\right)^{-1}-I\right\|<1
$$

where $I$ is the identity matrix. Condition (13) imposes certain restrictions on the choice of the approximate model used in inversion and consequently in the design. In the particular case of a completely decoupled MIMO system, the condition (13) is equivalent to:

$$
\begin{aligned}
\operatorname{sgn}\left(\frac{\partial g_{i}}{\partial u_{i}}\right) & =\operatorname{sgn}\left(\frac{\partial \hat{g}_{i}}{\partial u_{i}}\right), i=1, \cdots, m \\
\left|\frac{\partial \hat{g}_{i}}{\partial u_{i}}\right| & >\left|\frac{\partial g_{i}}{\partial u_{i}}\right| / 2>0, i=1, \cdots, m
\end{aligned}
$$

Control System Architecture. The main components are the model inversion/linearization block identified 
by $\hat{g}^{-1}(y, u)$ and the adaptive neural network based element designed to cancel the effect of $\Delta$.

The input into the controlled system is the reference tracking $y_{c}$ and its $r$-th derivative $y_{c}^{(r)}$ generated by a reference model forced by an external input. The linear compensator has two outputs $\left(\nu_{d c}, \tilde{y}_{a d}\right) . \nu_{d c}$ is designed to stabilize the linearized system conceived as $y^{(r)}=\nu$. The second output $\tilde{y}_{a d}$ is a linear combination of the compensator states and its input $(\tilde{y})$. This signal will be constructed to ensure an implementable error signal that is used to adapt the NN weights.

Tracking Error Analysis. Considering (8) and (10) leads to $\tilde{y}^{r}=-\nu_{d c}+\nu_{a d}-\Delta$. For the case $\Delta=0$, the adaptive term $\nu_{a d}$ is not required and the error dynamics reduce to

$$
\tilde{y}^{r}=-\nu_{d c}
$$

Design of the Linear Compensator. Calling $e_{i}=$ $\left\{\tilde{y}_{i}, \dot{y}_{i} \cdots, \tilde{y}_{i}^{\left(r_{i}-1\right)}\right\}^{\top}$ for $i=1, \cdots, m$ and denoting $e=\left\{e_{1}^{\top}, e_{2}^{\top}, \cdots, e_{m}^{\top},\right\}^{\top}$ the linear error dynamics can be stated as (15) with $\tilde{y}=C e$ where

$$
C=\left[\begin{array}{cccc}
c_{1} & 0 & \cdots & 0 \\
0 & c_{2} & \cdots & 0 \\
\vdots & \vdots & \ddots & \\
0 & 0 & \cdots & c_{m}
\end{array}\right], c_{i}=\left[\begin{array}{llll}
1 & 0 & \cdots & 0
\end{array}\right]
$$

$C$ is $m \times\left(r_{1}+\cdots+r_{m}\right)$ and $c_{i}$ is $1 \times r_{i}$. The linearized plant is:

$$
\begin{aligned}
& \dot{x}=A x+B u \\
& y=C x
\end{aligned}
$$

where $A=\operatorname{diag}\left(A_{1}, A_{2}, \cdots, A_{m}\right), B=\left[B_{1}^{\top} B_{2}^{\top} \cdots\right.$ $\left.B_{m}^{\top}\right]^{\top}$ and

$$
\begin{aligned}
& A_{i}=\left[\begin{array}{ccccc}
0 & 1 & 0 & \cdots & 0 \\
0 & 0 & 1 & \cdots & 0 \\
\vdots & \vdots & \vdots & \ddots & \vdots \\
0 & 0 & 0 & \cdots & 1 \\
0 & 0 & 0 & \cdots & 0
\end{array}\right], B_{1}=\left[\begin{array}{ccc}
0 & \cdots & 0 \\
\vdots & \ddots & \vdots \\
1 & \cdots & 0
\end{array}\right] \\
& B_{i}=\left[\begin{array}{ccccc}
0 & 0 & \cdots & 0 & 0 \\
\vdots & \vdots & \vdots & \ddots & \vdots \\
0 & \cdots & 1 & \cdots & 0
\end{array}\right], B_{m}=\left[\begin{array}{ccc}
0 & \cdots & 0 \\
\vdots & \ddots & \vdots \\
0 & \cdots & 1
\end{array}\right]
\end{aligned}
$$

$A_{i}$ is $r_{i} \times r_{i}, B$ is $\left(r_{1}+\cdots+r_{m}\right) \times m, B_{i}$ is $r_{i} \times m$, $i=1 \cdots m$. The transfer function is:

$$
G_{p}(s)=C(s I-A)^{-1} B=\frac{N_{p}(s)}{D_{p}(s)}
$$

$N_{p}(s)$ is the numerator matrix and $D_{p}(s)$ is the denominator polinomial. The $m$-input, $2 \times m$-output transfer matrix of the linear compensator is

$$
\left\{\begin{array}{l}
\nu_{d c}(s) \\
\tilde{y}_{a_{d}}(s)
\end{array}\right\}=\frac{1}{D_{d c}(s)}\left\{\begin{array}{l}
N_{d c}(s) \\
N_{a d}(s)
\end{array}\right\} \tilde{y}(s)
$$

where $s$ represents the complex Laplace variable, $N_{d c}(s)$ and $N_{a d}(s)$ are $m \times m$ transfer matrices and $D_{d c}(s)$ is the Hurwitz compensator characteristic polinomial.
Assumption 3 The linearized system (20) is stabilized using a stable linear dynamic compensator. Since the error dynamics consist of $r$ pure integrators the closed loop transfer function of the system is given by

$$
\begin{aligned}
\tilde{y}_{a d}(s)= & {\left[s^{r} D_{d c}(s)+N_{d c}(s) N_{p}(s)\right]^{-1} } \\
& N_{a d}(s)\left(\nu_{a d}-\Delta\right)(s)
\end{aligned}
$$

According to Routh-Hurwitz stability criterion, as a necessary closed loop stability condition, the characteristic polinomial of (22) should accomplish:

$$
q \equiv \operatorname{deg}\left(D_{d c}\right) \geq \operatorname{deg}\left(N_{d c} N_{p}(s)\right) \geq r-1
$$

This dictates the design of the linear dynamic compensator.

Neural Network Based Approximation. Linearly parameterized neural networks

$$
y=W^{\top} \phi(x)
$$

are universal approximators, where $\phi(\cdot)$ can be selected as a basis over the domain of approximation as for example

$$
\phi(x)=\left\{\phi_{i}(x)\right\}=\left\{e^{\left(x-x_{0 i}\right)^{\top}\left(x-x_{0 i}\right) / \sigma^{2}}\right\}
$$

in which $x_{0 i}$ are the centers and $\sigma$ is a suitable dispersion constant. Then a general function $f(x) \in \mathcal{C}^{k}$, $x \in D \subset \mathbb{R}^{n}$ can be written as:

$$
f(x)=W^{\top} \phi(x)+\epsilon(x)
$$

where $\epsilon(x)$ is the functional reconstruction error. In general, given a constant real number $\epsilon_{o}>0, f(x)$ is within $\epsilon_{o}$ range of the $\mathrm{NN}$, if there exist constant weights $W$, such that for all $x \in \mathbb{R}^{n}$ equation (26) holds with $\|\epsilon\|<\epsilon_{o}$.

Definition 1 The functional range of $N N(24)$ is dense over a compact domain $x \in \mathcal{D}$, if for any $f(\cdot) \in \mathcal{C}^{k}$ and $\epsilon_{o}$ there exists a finite set of bounded weights $W$, such that (26) holds with $\|\epsilon\|<\epsilon_{o}$.

Various publications prove this assertion (Sanner and Slotine, 1992) The following theorem extends these results to map the unknown dynamics of an observable plant form available input/output history. A proof can be found in (Calise, Hovakimyan and Idam, 2001).

Theorem 1 Given $\epsilon>0$, there exists a set of bounded ideal weights $W$ such that $\Delta(x, y, \nu)$, associated with system (1), can be approximated over a compact domain $\mathcal{D} \subset \Omega \times \mathbb{R}$ by a linearly parameterized neural network

$$
\Delta=W^{\top} \phi(\eta)+\epsilon(\eta),\|\epsilon\|<\epsilon_{o}
$$

using the input vector

$$
\eta(t)=\left[\begin{array}{lll}
1 & \bar{\nu}_{d}^{\top}(t) & \bar{y}_{d}^{\top}(t)
\end{array}\right]^{\top}
$$

where

$$
\begin{aligned}
& \bar{\nu}_{d}^{\top}(t)=\left[\begin{array}{llll}
\nu(t) & \nu(t-d) & \cdots & \nu\left(t-\left(n_{1}-r-1\right) d\right)
\end{array}\right]^{\top} \\
& \bar{y}_{d}^{\top}(t)=\left[\begin{array}{llll}
y(t) & y(t-d) & \cdots & y\left(t-\left(n_{1}-1\right) d\right)
\end{array}\right]^{\top}
\end{aligned}
$$

with $n_{1} \geq n$ and $d>0$, if there exists a suitable basis of functions $\phi(\cdot)$ on the compact domain $\mathcal{D}$. 
The output of the adaptive element is designed as

$$
\nu_{a d}=\hat{W}^{\top} \phi(\eta)
$$

where $\hat{W}$ are the estimates of the ideal weights $W$.

Assumption 4 There exists a fixed point solution to

$$
\nu_{a d}=W^{\top} \phi\left(\cdots, \nu_{a d}\right)
$$

over the entire input domain of interest.

Construction of SPR Transfer Functions. According to (22), $\tilde{y}_{a d}$ will be used to construct the rule for adapting $\hat{W}$ in (31). Using (27) and (31) in (22) gives,

$$
\tilde{y}_{a d}(s)=G(s)\left(\tilde{W}^{\top} \phi(\eta)-\epsilon\right)
$$

where $\tilde{W}=\hat{W}-W$. For the $\mathrm{NN}$ adaptation rule to be realizable, i. e. dependent on available data only, the transfer function $G(s)$ must be strictly positive real (SPR). However, the relative degree of $G(s)$ is at last $r$. If $r>1, G(s)$ cannot be SPR. If $r=1, G(s)$ can be made SPR by a proper construction of $N_{a d}(s)$. To achieve $G(s)$ SPR in the $r>1$ case, a stable low pass filter $T^{-1}(s)$ is introduced (Kim and Lewis, 1998) in (32) as

$$
\tilde{y}_{a d}(s)=G(s) T(s)\left(\tilde{W}^{\top} \phi_{f}(\eta)+\delta-\epsilon_{f}\right)(s)
$$

where $\phi_{f}$ and $\epsilon_{f}$ are the signals $\phi$ and $\epsilon$ after being filtered through $T^{-1}(s)$ and $\delta$ is the so called mismatch term given by

$$
\delta(s)=T^{-1}(s)\left(W^{\top} \phi\right)-\tilde{W}^{\top} \phi_{f}
$$

The numerator of the transfer function $G(s) T(s)$ in (33) is $T(s) N_{a d}(s)$. The polynomial $T(s)$ is Hurwitz, but otherwise can be freely chosen, along with the numerator polynomial $N_{a d}(s)$. Hence the product $G(s) T(s)$ can be chosen to make it SPR.

Neural Network Adaptation Rule. As can be seen in (33) the filter $T^{-1}(s)$ should operate on all the components of the NN matrix $\phi$. All these filters can be cast in a state space realization

$$
\begin{aligned}
& \dot{z}_{f}=A_{f} z_{f}+B_{f} \phi \\
& \phi_{f}=C_{f} z_{f}
\end{aligned}
$$

Since the filter is stable, $\exists P_{f} \succ 0$, satisfying

$$
A_{f}^{\top} P_{f}+P_{f} A_{f}=-Q_{f}
$$

for any $Q_{f} \succ 0$. The signals $\phi_{f}$ are used in the following adaptation rule

$$
\dot{\hat{W}}=-\Gamma_{W}\left(\tilde{y}_{a d} \phi_{f}+\lambda_{W} \hat{W}\right)
$$

where $\Gamma_{W} \succ 0$ and $\lambda_{W}>0$ are the adaptation gains.

\section{BOUNDEDNESS STATEMENT}

The following theorem establishes sufficient conditions for boundedness of the error signals and neural network weights in the proposed closed-loop adaptive output feedback architecture.
Theorem 1 Subject to assumptions 1-3, the error signal of the system comprised of the dynamics in (1), together with the dynamics associated with the realization of the controller in (5) and the NN adaptation rule in (37), are uniformly ultimately bounded, provided that the following conditions hold

$$
Q_{m}>\left\|C_{c l}\right\|, \lambda_{W}>c^{2} / 4
$$

where $Q_{m}$ is the minimum eigenvalue of $Q$. If $G(s) T(s)$ is SPR then (33) can be represented as

$$
\begin{aligned}
\dot{z} & =A_{s} z+B_{s}\left(\tilde{W}^{\top} \phi_{f}+\delta-\epsilon_{f}\right) \\
\tilde{y}_{a d} & =C_{s} z
\end{aligned}
$$

and complies with the Lefschetz-Kalman-Yakubovitz Lemma i. e. there exists $Q \succ 0$ such that the solution $P$ of

$$
A_{s}^{\top} P+P A_{s}^{\top}=Q
$$

is positive definite and $P B_{s}=C_{s}^{\top}$. Consider now the positive function

$$
L=\frac{1}{2} z^{\top} P z+\frac{1}{2} z_{f}^{\top} P_{f} z_{f}+\frac{1}{2}\left(\tilde{W}^{\top} F^{-1} \tilde{W}\right)
$$

where $F \succ 0 . P \succ 0$ and $P_{f} \succ 0$ are solutions of (40) and (36) for some $Q \succ 0$ and $Q_{f} \succ 0$. By incorporating the adaptation rule (37), the derivative of (41) gives:

$$
\begin{gathered}
\dot{L}=-z^{\top} Q z+\tilde{y}_{a d}\left(\delta-\epsilon_{f}\right)-z_{f}^{\top} Q_{f} z_{f}+ \\
z_{f}^{\top} P_{f} B_{f} \phi-\lambda_{W} \tilde{W}^{\top}(\tilde{W}+W)
\end{gathered}
$$

From (34)

$$
\|\delta\| \leq c\|\tilde{W}\|_{F}, c>0
$$

where $\|\cdot\|_{F}$ indicates norm in the sense of Frobenius. Using (43) and (27), $\epsilon_{f}$ can be bounded as

$$
\left|\epsilon_{f}\right| \leq|\epsilon| \leq\left|\epsilon_{o}\right|
$$

Using this bound and (43), (42) can be bounded as:

$$
\begin{aligned}
\dot{L} \leq & -Q_{m}\|z\|^{2}+c\left\|\tilde{y}_{a d}\right\|\|\tilde{W}\|_{F}+\epsilon_{o}\left\|\tilde{y}_{a d}\right\| \\
& -Q_{f_{m}}\left\|z_{f}\right\|^{2}+\left\|P_{f} B_{f}\right\|\left\|z_{f}\right\|\|\phi\| \\
& -\lambda_{W}\|\tilde{W}\|_{F}\left(\|\tilde{W}\|_{F}-\|W\|_{F}\right)
\end{aligned}
$$

where $Q_{m}$ and $Q_{f}$ are the minimum eigenvalues of $Q$ and $Q_{f}$ respectively. For (39) $\left\|\tilde{y}_{a d}\right\| \leq\left\|C_{s}\right\|_{F}\|z\|$. Introducing $W_{o}$ is a bound for the ideal weights, according with $\|W\|_{F} \leq W_{o}$, completion of squares in (45) together with some standard algebraic manipulations and grouping leads to

$$
\begin{aligned}
\dot{L} \leq & -\left(\left\|\tilde{y}_{a d}\right\| \sqrt{\frac{Q_{m}}{\left\|C_{s}\right\|_{F}}}-1-\frac{1}{2} \frac{\epsilon_{o}}{\sqrt{Q_{m} /\left\|C_{s}\right\|_{F}-1}}\right)^{2} \\
& +\frac{1}{4} \frac{\epsilon_{o}^{2}}{Q_{m} /\left\|C_{s}\right\|_{F}-1}-\left(\left\|z_{f}\right\| \sqrt{Q_{f_{m}}}\right. \\
& \left.-\frac{1}{2} \frac{\left\|P_{f} B_{f}\right\|_{F}\|\phi\|}{\sqrt{Q_{f}}}\right)^{2} \frac{1}{4} \frac{\left\|P_{f} B_{f}\right\|_{F}^{2}\|\phi\|^{2}}{Q_{f_{m}}} \\
& -\left(\|\tilde{W}\|_{F} \sqrt{\lambda_{W}-c^{2} / 4}-\frac{1}{2} \frac{\lambda_{W} W_{o}}{\sqrt{\lambda_{W}-c^{2} / 4}}\right)^{2} \\
& +\frac{1}{4} \frac{\left(\lambda_{W} W_{o}\right)^{2}}{\lambda_{W}-c^{2} / 4}
\end{aligned}
$$


$\dot{L} \leq 0$ holds outside an ellipsoid in the space of the error variables $\tilde{y}_{a d}, z_{f}$ and $\tilde{W}$, given by setting the right hand side of (46) to zero. This ellipsoid touches the origin of the error space and this demonstrates uniform ultimate boundedness of the signals $\tilde{y}_{a d}, z_{f}$ and $\tilde{W}$. Since the transfer function between the tracking error $\tilde{y}$ and $\tilde{y}_{a d}$ is stable, boundedness of the latter guarantees that $\tilde{y}$ is also bounded.

\section{APPLICATION TO THE SCALE MODEL HELICOPTER}

Using Lagrangian formulation over a model helicopter restricted to a 2DOF platform (Avila, Brogliato, Dzul and Lozano, 2003) results in an under actuated 3DOF plant

$$
\begin{aligned}
M(q) \ddot{q}+C(q, \dot{q}) \dot{q}+G(q) & =Q(q, \dot{q}, u) \\
y & =h(q)
\end{aligned}
$$

where $q=(z, \phi, \gamma)^{\top}$ represents height, yaw angle and main rotor azimuth angle respectively. Also $h(q)=$ $(z, \phi)^{\top}$. The angle $\gamma$ will be considered as unmodelled dynamics. Initially $|\dot{\gamma}(0)| \geq g_{0}>0$ and $z(0) \leq L_{0}$, thus comprehending take-off and landing $(z(0) \leq$ $\left.L_{0}\right)$, and vertical flight (hover) $\left(z(0)<L_{0}\right)$

$$
\begin{aligned}
M(q) & =\left[\begin{array}{ccc}
c_{0} & 0 & 0 \\
0 & c_{1}+c_{2} \cos ^{2}\left(c_{3} \gamma\right) & c_{4} \\
0 & c_{4} & c_{5}
\end{array}\right] \\
C(q, \dot{q}) & =\left[\begin{array}{ccc}
0 & 0 & 0 \\
0 & c_{6} \sin \left(2 c_{3} \gamma\right) \dot{\gamma} & c_{6} \sin \left(2 c_{3} \gamma\right) \dot{\phi} \\
0 & -c_{6} \sin \left(2 c_{3} \gamma\right) \dot{\phi} & 0
\end{array}\right] \\
G(q) & \left.=\left[\begin{array}{c}
c_{7} \\
0 \\
0
\end{array}\right] \begin{array}{c} 
\\
c_{8} \dot{\gamma}^{2} u_{1}+c_{2} \dot{\gamma}+c_{10} \\
c_{11} \dot{\gamma}^{2} u_{2} \\
\left(c_{12} \dot{\gamma}+c_{13}\right) u_{1}+c_{14} \dot{\gamma}^{2}+c_{15}
\end{array}\right]
\end{aligned}
$$

The $c_{i}$ 's, $i=1, \cdots, 15$ are given in (Avila, Brogliato, Dzul and Lozano, 2003). The actuation $u=\left(u_{1}, u_{2}\right)^{\top}$ is saturated so $u_{0}^{-} \leq u \leq u_{0}^{+}$with $u_{0}^{-}=$ $(-0.002,-0.002)^{\top}$ and $u_{0}^{+}=(0,0.002)^{\top} \cdot M(q)$ is invertible so including the one sided smooth constraint (Avila, Brogliato, Dzul and Lozano, 2003)

$$
\begin{aligned}
\ddot{q}= & M^{-1}(q)(-C(q, \dot{q}) \dot{q}-G(q)+Q(q, \dot{q}, u)-\Delta g \lambda) \\
y= & h(q) \\
& z \leq L_{0}, \lambda \geq 0, \lambda\left(z-L_{0}\right)=0 \\
& \Delta g=\frac{\partial z}{\partial q}=[1,0,0]^{\top}
\end{aligned}
$$

The plant (49) when $z-L_{0}<0$ has vector relative degree $\mathbf{r}=(2,2)$. The adopted plant model for control purposes is:

$\dot{x}=\left(\begin{array}{cccc}0 & 0 & 1 & 0 \\ 0 & 0 & 0 & 1 \\ 0 & 0 & 0 & 0 \\ 0 & 0 & 0 & 0\end{array}\right) x+\left(\begin{array}{cc}0 & 0 \\ 0 & 0 \\ b_{0} & 0 \\ 0 & -b_{0}\end{array}\right) u, y=\left(\begin{array}{llll}1 & 0 & 0 & 0 \\ 0 & 1 & 0 & 0\end{array}\right) x$ which gives

$$
G_{p}(s)=\frac{b_{0}}{s^{2}}\left(\begin{array}{cc}
1 & 0 \\
0 & -1
\end{array}\right)
$$

Drift values for the linear controller are: $u_{10}=$ $-0.46 \times 10^{-4}, u_{20}=0, g_{0}=-124.6[\mathrm{rad} / \mathrm{s}]$. Feedback linearization in (4) has been performed with the transformation $\hat{g}=u$. A first order lead compensator structure was selected to stabilize the associated error dynamics and also the SPR condition

$$
\begin{aligned}
\nu_{d c}(s) & =k_{c} \frac{(s / \alpha+1)}{(s / \beta+1)}\left(\begin{array}{cc}
1 & 0 \\
0 & -1
\end{array}\right) \\
\tilde{y}_{a d}(s) & =k_{o} \frac{\left(s / \alpha_{o}+1\right)}{(s / \beta+1)}\left(\begin{array}{cc}
1 & 0 \\
0 & -1
\end{array}\right) \\
T^{-1}(s) & =\frac{1}{s+1}
\end{aligned}
$$

with $\alpha \approx 0.1, \beta \gg \alpha, b_{0}=10^{4}, k_{c} \approx 1.25 / b_{0}$ A second order command filter was implemented for each of the output channels so that

$$
y_{c}(s)=\frac{\omega_{n}^{2}}{s^{2}+2 \zeta \omega_{n} s+\omega_{n}^{2}} y_{r e f}(s)
$$

with $\omega_{n}=3, \zeta=2$. The network has $8 \times 2$ neurons having (25) as activating function. The weights where randomly generated and the adaptation gains are: $\Gamma_{W}=50, \lambda_{W}=1$. The pure lag considered in (29) is $d=0.5 \mathrm{~s}$.

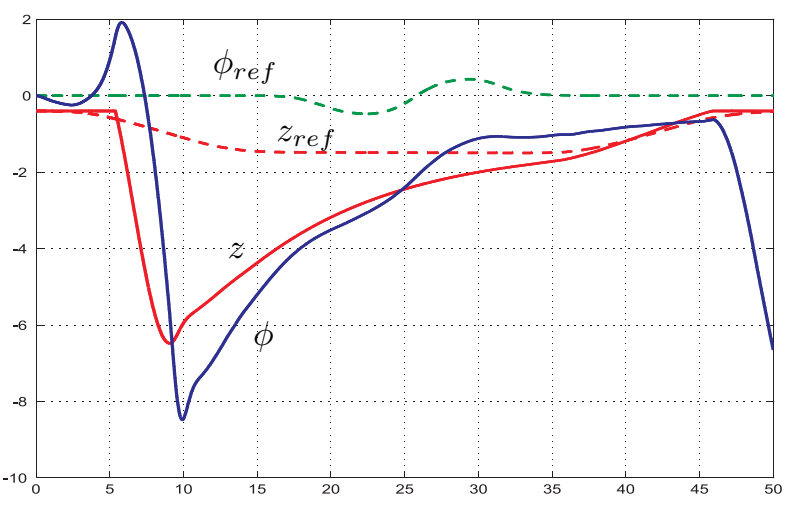

Fig. 1. Tracking without adaptation

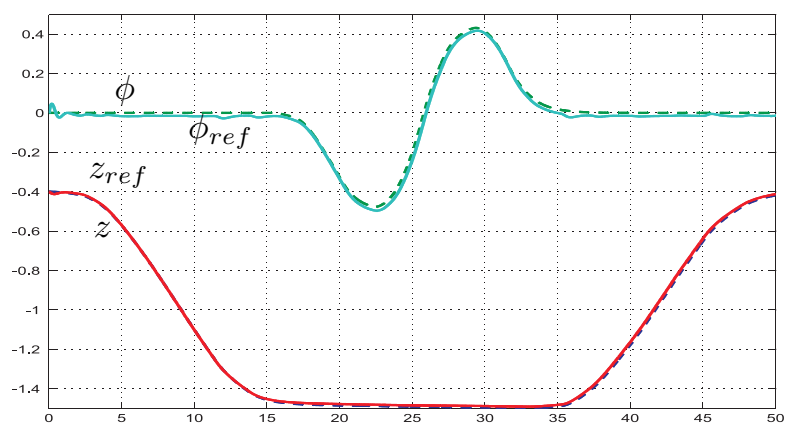

Fig. 2. Tracking with adaptation 


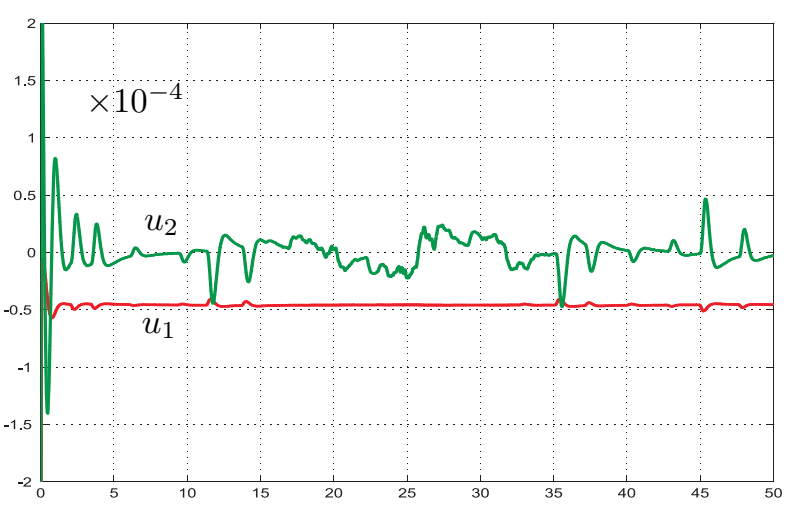

Fig. 3. Control with adaptation

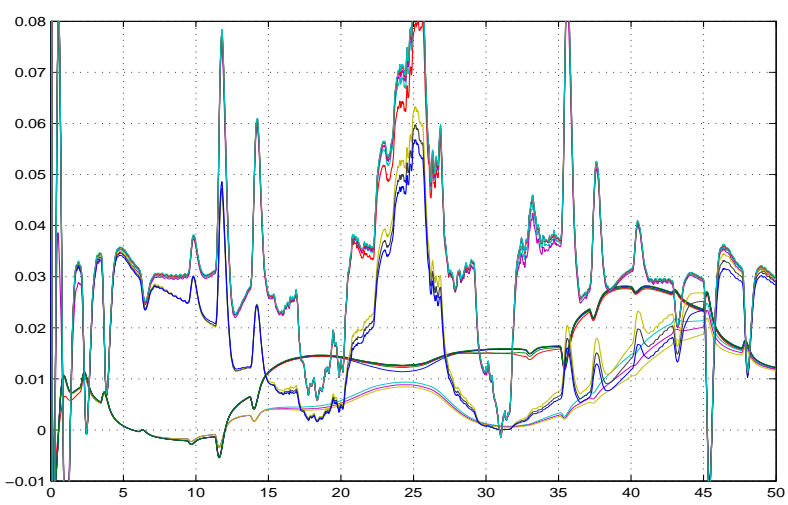

Fig. 4. Neural Network weights evolution

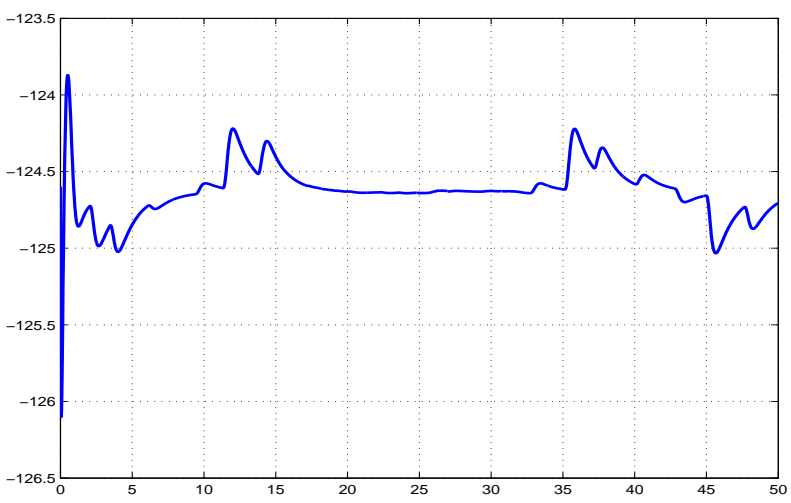

Fig. 5. Main rotor rotation

In Fig. 1 appears the linear dynamic compensation performance, without adaptation. References are dotted and outputs are continuous lines. Adaptation can be seen in Fig. 2 where tracking errors are maintained bounded all over the maneuvers: take off $(z \leq$ $\left.L_{0}\right) 0<t \leq 15$, hovering $\left(z<L_{0}\right) 15<t \leq 35$ and landing $\left(z \leq L_{0}\right) 35<t \leq 50$. Control history is shown in Fig. 3. The radial basis neural network adapt quite well to cancel the inversion residuals even when the constraint is active. At the beginning the adaptation effort is stronger (Fig. 4), producing undesirable transients. This could be mitigated preadapting the initial weights. Finally in Fig. 5 appears the main rotor rotation that can be considered as unmodelled dynamics.

\section{CONCLUSIONS}

The presented technique can be used successfully in MIMO plants with poor modelling. Under the assumption that the plant has well defined vector relative degree, a radial basis neural network is introduced to cancel the inversion error. The network learns on line and no off line training is required. No state estimation is needed and the full dimension of the plant and its internal dynamics may be unknown or poorly modelled. The method is applicable to systems of unknown but bounded dimension, being adaptive to both parametric uncertainty and unmodelled dynamics. We are currently developing the application of this technique to the MARVIN (C. Deg, M. Musial and G. Hommel, 2004) helicopter.

\section{REFERENCES}

Avila, J.C., Brogliato, B, Dzul, A. and Lozano, R. (2003). Nonlinear modelling and control of helicopters. Automatica 39(9), 1583-1596.

Calise, A.J., Hovakimyan, N. and Idam, M. (2001). Adaptive Output Feedback Control of Nonlinear Systems using Neural Networks. Automatica (Special Issue on Neural Networks for Feedback Control) 37(8), 1201-1211.

Ge, S.S., Hang, C.C. and Zhang, T. (1999). Adaptive Neural Network Control of Nonlinear Systems by State and Output Feedback. IEEE Transactions on Systems, Man and Cybernetics 29(6), 818-828.

Hovakimyan, N. and Calise, J. (2002). Adaptive Output Feedback Control of Uncertain MultiInput Multi-Output Systems using Single Hidden Layer Neural Networks. American Control Conference, 2002.

C. Deg, M. Musial and G. Hommel (2004). Control and Simulationh of an Autonomously Flying Model Helicopter. IAV 2004.

Kim, Y. and Lewis, F. (1998). High Level Feedback Control with Neural Networks. World Scientific, NJ.

Sanner, R. and Slotine, J. (1992). Gaussian networks for direct adaptive control. IEEE Transactions on Neural Networks 3(6), 837-864.

Seshagiri, S., Khalil, H.K. (2000). Output Feedback Control of Nonlinear Systems Using RBF Neural Networks IEEE Transactions on Neural Networks 11(1), 69-79. 